\title{
A Comparative Study of Optical and Magnetic Properties of Undoped and Cobalt Doped Manganese Oxide Nano Particles
}

\author{
S Karpagavalli ${ }^{1}$, S John Kennady Vethanathan ${ }^{2}$, S Perumal $^{3}$, \\ D Priscilla Koilpillai ${ }^{4}$, A Suganthi ${ }^{5}$ \\ ${ }^{1 \& 5}$ Department of Physics, Govindammal Aditanar College for women, Tiruchendur-628 215 Tamil Nadu, India \\ ${ }^{2 \& 4}$ Department of Physics, St. Johns College, Palayamkottai- 627002 Tamil Nadu, India \\ ${ }^{3}$ Department of Physics, Noorul Islam College of Arts and Science, Kumaracoil-629180 Tamil Nadu, India
}

\begin{abstract}
Manganese oxide and Cobalt doped Manganese oxide nanoparticles are synthesized by solvothermal route using ethylene glycol as a solvent. The structural investigations are done by X-ray diffraction (XRD). The average grain size and lattice parameters are calculated. The particle size is confirmed by scanning electron microscope (SEM) analysis. The functional groups exists in the material are construed by Fourier transform infrared (FTIR) spectral analysis. The chemical composition and purity of the samples are inspected by using Energy-dispersive X-ray spectral analysis (EDAX).The optical properties are analyzed by Ultraviolet-Visible (UV-Vis) spectroscopy. A UV-Vis spectra shows that Cobalt doped Manganese oxide nanoparticles acquires blue shift. The magnetic properties of all the samples are reported using vibrating sample magnetometer (VSM) at room temperature. The values of saturation magnetization, retentivity, coercivity and squareness ratio are obtained from the magnetic studies.
\end{abstract}

Keywords: EDAX, FTIR, Solvo thermal method, UV-Vis, VSM and XRD

\section{Introduction}

Materials innovation and materials fabrication are at the heart of nanoscience and engineering. Nanoparticles are key focus of research for a wide novel application. At nanolevel the properties are greatly changed, as the size of the particles changed owing to the wide spread applications. Among nano materials magnetic nanoparticles are of extreme interest to researchers according to their very good magnetic properties. Magnetic nanoparticles have a wide range of applications, including magnetic fluids recording, catalysis, photo catalysis, electrochemical microwave absorption, magnetic resonance imaging, medical diagnosis, data storage and environmental remediation and as an electrode for supercapacitors and lithium ion batteries (LIB) [1].The magnetic behavior of manganese oxide nanoparticles are of increasing research interest due to their large value of intrinsic magnetic moment [2]. Manganese oxides have attracted considerable interest as inexpensive and non-toxic alternative materials as rechargeable battery cathodes. The Cobalt doped Manganese oxide nano particles exhibited clear hysteresis showing typical ferromagnetic behavior, shows that the material formed is of soft magnetic material which is useful in magnetic memory devices. Various methods have been used to prepare nanoparticles. But, Microwave assisted solvothermal method is a chemical method that use microwave radiation for heating materials containing electrical charges for instance polar molecule in the solvent or charge ion in the solid. As compared to the other heating methods microwave assisted solution fabrication methods have got more focus of research because of rapid processing, high reaction rate, reduce reaction time and high yield of product [3].

In the present work, nanocrystalline particles of $\mathrm{MnO}_{2}$ and Cobalt doped $\mathrm{MnO}_{2}$ are prepared by solvothermal method. The synthesized nanoparticles are characterized by XRD, SEM, EDAX, FTIR, UV-VISIBLE and VSM.

\subsection{Synthesis:}

\section{Materials and Methods}

To prepare pure Manganese oxide nanoparticles, AR grade manganese acetate tetra hydrate [Mn $\left.\left(\mathrm{CH}_{3} \mathrm{CO}\right)_{2} .4 \mathrm{H}_{2} \mathrm{O}\right]$ and urea act as a catalyst was dissolved in ethylene glycol. The mixed solution was stirred well for 3 hour. The completely dissolved solution was kept in a domestic microwave oven. The microwave irradiation was carried out till the solvent evaporates completely. The prepared samples were washed four or five times with distilled water. Then the sample was washed with acetone to remove unwanted organic impurities present. The synthesized nanoparticles are filtered and dried in an oven at $323 \mathrm{~K}$. As a result of annealing to a high temperature $580 \mathrm{~K}$ in a muffle furnace for $5 \mathrm{~h}$, the transition metal Manganese converted into their oxides to get pure Manganese Oxide nanoparticles. 
To prepare Cobalt doped manganese oxide nanoparticles, analytical grade manganese acetate tetrahydrate $\left[\mathrm{Mn}\left(\mathrm{CH}_{3} \mathrm{CO}\right)_{2} .4 \mathrm{H}_{2} \mathrm{O}\right]$, Cobalt acetate dihydrate $\left[\mathrm{Co}\left(\mathrm{CH}_{3} \mathrm{CO}\right)_{2} \cdot 2 \mathrm{H}_{2} \mathrm{O}\right]$ and urea $\left[\mathrm{CH}_{4} \mathrm{~N}_{2} \mathrm{O}\right]$ are used as starting materials was dissolved in ethylene glycol ( i.e.,) $\mathrm{Mn}_{\mathrm{x}} \mathrm{Co}_{1-\mathrm{x}} \mathrm{O}(\mathrm{x}=0.98,0.96,0.94$ and 0.92$)$ The precipitates were washed four or five times with distilled water and acetone to eliminate other impurities. The synthesized nanoparticles are filtered and dried in an oven at $323 \mathrm{~K}$. On heating to a high temperature $580 \mathrm{~K}$ in a muffle furnace for $5 \mathrm{~h}$ to obtain Cobalt doped Manganese Oxide nanoparticles.

Compare the influence of dopant Cobalt 2\%, 4\%, $6 \%$ and $8 \%$ concentration on the structural, optical and magnetic properties with pure Manganese oxide nanoparticles.

\subsection{Characterization Techniques:}

The structural characteristics of formed $\mathrm{MnO}_{2}$ and cobalt doped $\mathrm{MnO}_{2}$ (2\%,4\%,6\% and 8\%) nanoparticles are determined by the aid of X-ray diffraction (XRD) (Bruker AXS D8 Advance model diffractometer using $\mathrm{CuK} \alpha . \lambda=0.15406 \mathrm{~nm}$ radiation). The morphology of the as-prepared sample is examined by Scanning electron microscope (SEM) (JEOL Model JSM - 6390LV). The functional groups and other impurities present in the material are interpreted by Fourier Transform Infrared spectroscopy (FTIR) (SHIMADZU MODEL -IR AFFINITY -1) in the wavelength range 400-4000 $\mathrm{cm}^{-1}$. It is an added confirmation for the purity of the samples by using Energy-dispersive X-ray spectroscopy (EDAX). The optical properties are analyzed by Ultraviolet -Visible spectrometer (Model: Varian, Cary 5000) in the wavelength range 200-1000 $\mathrm{cm}^{-1}$. Magnetic study is done by using vibrating sample magnetometer (VSM). (Model: LakeShore 7410).

\subsection{X-Ray Diffraction Analysis:}

\section{Results and Discussion}

The XRD patterns of pure and cobalt $\left(2 \%, 4 \%, 6 \%\right.$ and $8 \%$ ) doped $\mathrm{MnO}_{2}$ nanoparticles are presented in Fig1.Diffraction patterns reveal that all the samples are of crystalline nature. All the diffraction peaks are well indexed to the orthorhombic structure $\alpha=\beta=\gamma=90^{\circ}$. The crystallite size was estimated using Debye Scherrer's equation;

$$
D=k \lambda / \beta \operatorname{Cos} \theta(\mathrm{m})
$$

Where, $\mathrm{k}$ - Shape factor $\approx 0.9, \lambda$-Wavelength of the X-ray source $\left(1.5406 \mathrm{~A}^{0}\right)$ and $\beta$ - Full width half maximum (FWHM) of a diffraction peak

The lattice constants $\mathrm{a}, \mathrm{b}, \mathrm{c}$ and the volumes are evaluated from XRDA software and the estimated values are recorded in Table 1
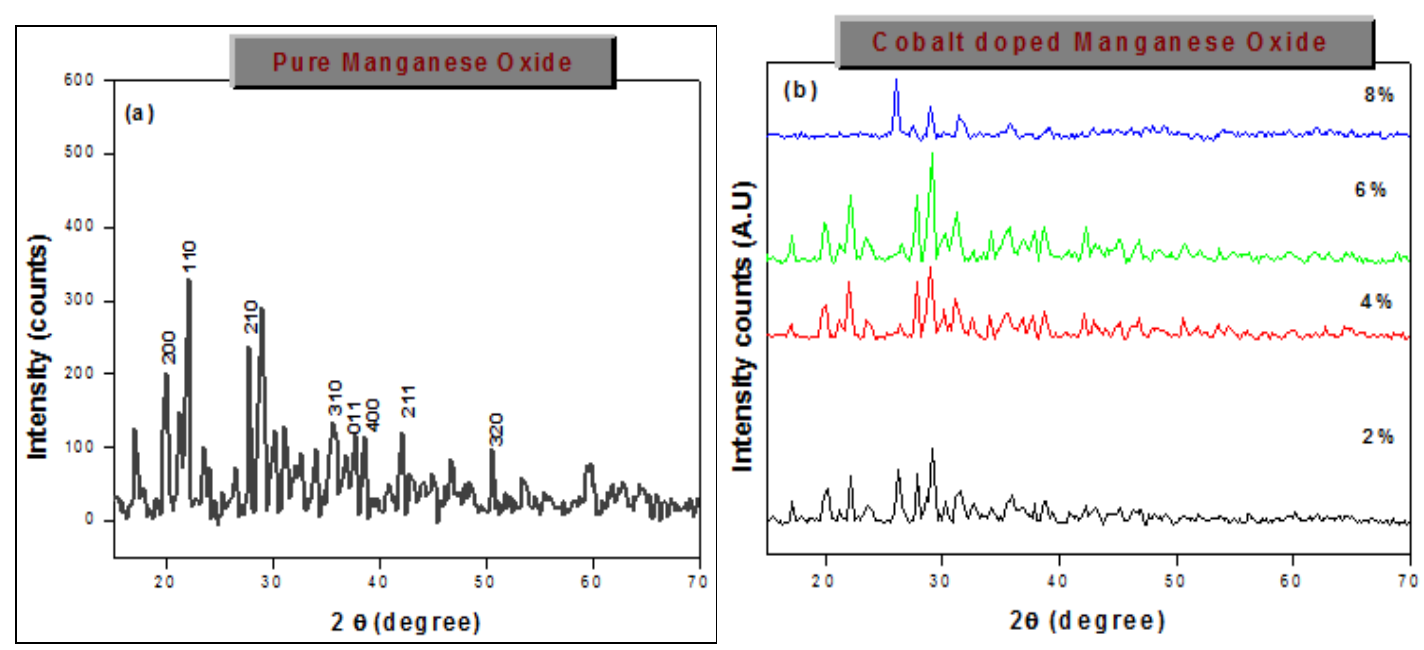

Fig.1 - XRD spectrum of (a) Pure Manganese Oxide (b) Cobalt doped Manganese Oxide nanoparticles

Table1: The Variation Of Particle Size And Lattice Parameters Of $\mathrm{MnO}_{2}$ : Co Nanoparticles Sample \begin{tabular}{l|l} 
Crystallite size & Lattice parameter $\left(\mathbf{A}^{0}\right)$ \\
\hline
\end{tabular} Volume

National Conference on Current Advancements in Physics $3^{\text {rd }} \& 4^{\text {th }}$ February 2017

Department of Physics, St. John's College, Palayamkottai-627 002, Tamilnadu, India. DOI 10.9790/4861-17002013442 
A Comparative Study of Optical and Magnetic Properties of Undoped and Cobalt Doped Manganese..

\begin{tabular}{|l|l|l|l|l|l|}
\hline & $(\mathrm{nm})$ & a & b & c & \\
\hline $\mathrm{MnO}_{2}$ & 39.4 & 9.2772 & 4.4900 & 2.8221 & 117.553 \\
\hline $\mathrm{MnO}_{2}: \mathrm{Co}(\mathbf{2} \%)$ & 36.2 & 9.2558 & 4.4870 & 2.8229 & 117.237 \\
\hline $\mathrm{MnO}_{2}: \mathbf{C o}(\mathbf{4} \%)$ & 34.7 & 9.1703 & 4.5673 & 2.7747 & 117.481 \\
\hline $\mathrm{MnO}_{2}: \mathbf{C o}(\mathbf{6} \%)$ & 32.8 & 9.3049 & 4.5035 & 2.8188 & 118.120 \\
\hline $\mathrm{MnO}_{2}: \mathbf{C o}(\mathbf{8} \%)$ & 32.1 & 9.1747 & 4.4733 & 2.8614 & 117.435 \\
\hline
\end{tabular}

\subsection{Scanning Electron microscope analysis:}

The SEM is widely used to identify phases based on qualitative chemical analysis and crystalline structure. Backscattered electron images can be used for rapid discrimination of phases in multiphase samples. The SEM analysis was carried out to investigate the detailed morphology of the synthesized pure $\mathrm{MnO}_{2}$ and cobalt doped $\mathrm{MnO}_{2}$ nanoparticles with different dopant Concentrations (2\%, 4\%, 6\% and 8\%) are shown in Fig 2.
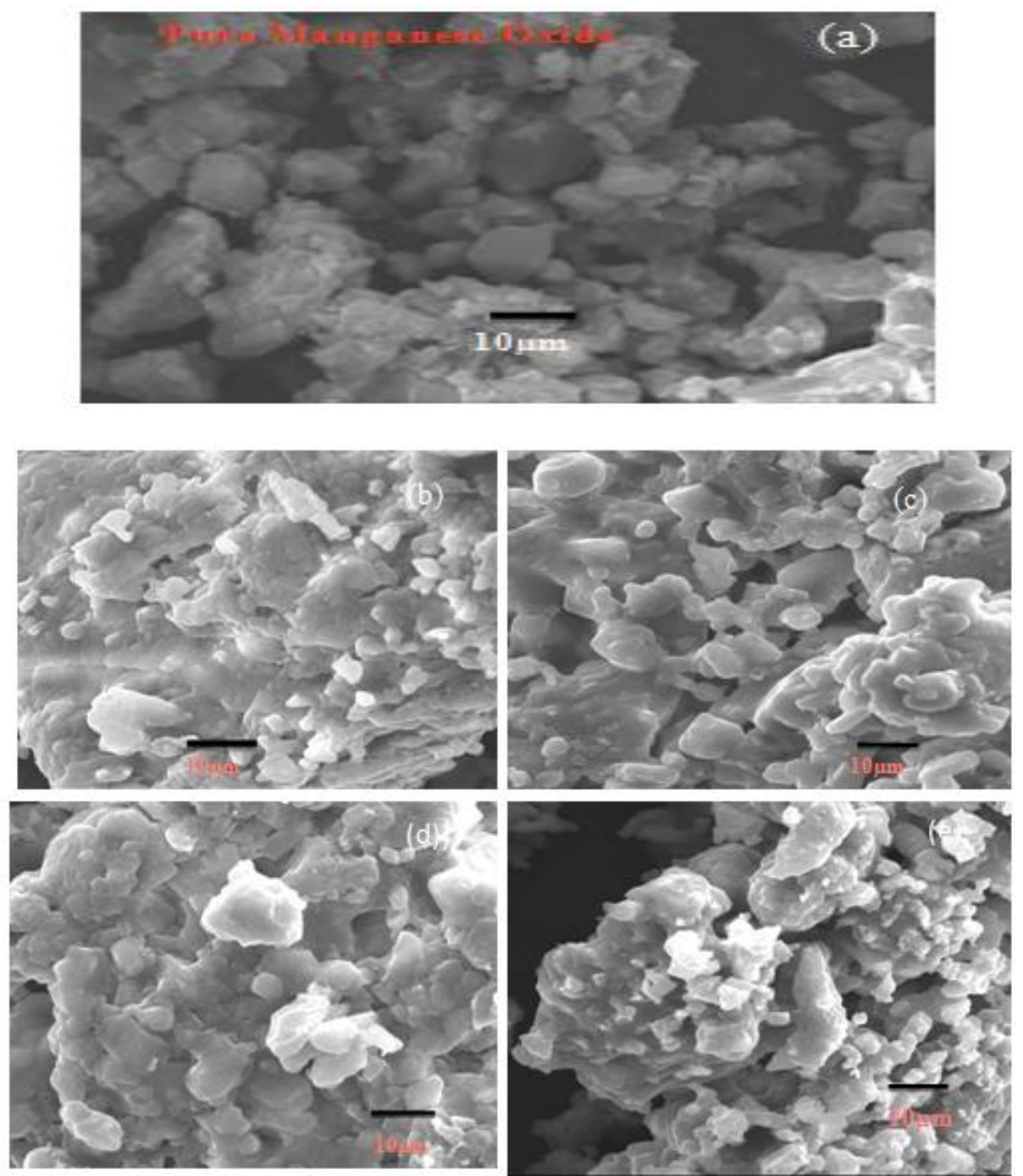

Fig.2 - SEM images of (a) Pure Manganese Oxide (b) 2\% Cobalt doped Manganese Oxide, (c) 4\% Cobalt doped Manganese Oxide, (d) 6\% Cobalt doped Manganese Oxide \& (e) $8 \%$ Cobalt doped Manganese Oxide

\subsection{Fourier transform infrared spectral analysis:}

FTIR spectrum was used to identify functional groups and other impurities present in the $\mathrm{MnO}_{2}$ and Cobalt doped $\mathrm{MnO}_{2}$ nano system with dopant concentration $(2 \%, 4 \%, 6 \%$ and $8 \%)$ is presented in Fig3. FTIR spectra of undoped and different levels of Cobalt incorporated $\mathrm{MnO}_{2}$ nano crystals are recorded in the range $4000-400$ $\mathrm{cm}^{-1}$. 

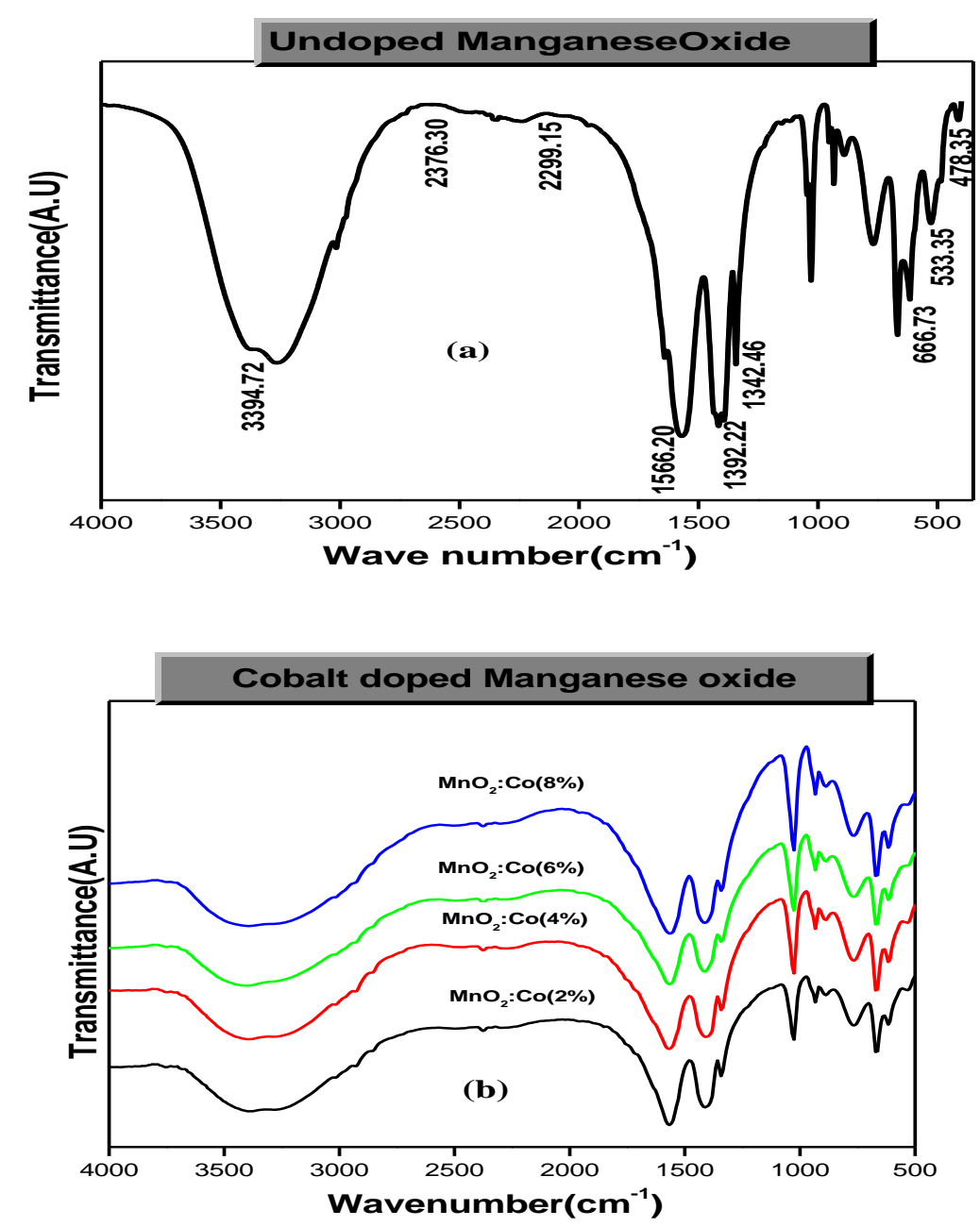

Fig.3- FTIR spectrum of (a) Pure Manganese Oxide and (b) Cobalt doped Manganese Oxide

\subsection{Energy-dispersive $\mathrm{X}$-ray spectral analysis (EDAX):}

Quantitative analysis of Cobalt $(2 \%, 4 \%, 6 \% \& 8 \%)$ doped $\mathrm{MnO}_{2}$ is put down in the pie chart.It is seen that no impurities are found out. Energy dispersive X-ray spectroscopy (EDAX) is an analytical technique used for the elemental analysis or chemical characterization of a sample. It relies on an interaction of some source of $\mathrm{X}$-ray excitation and a sample. Its characterization capabilities are due to the fundamental principle that each element has a unique atomic structure allowing unique set of peaks on its X-ray emission spectrum. The emission of characteristics X-rays from a specimen, a high energy beam of charged particles such as electron or protons or a beam of X-rays, is focused into the sample being studied.

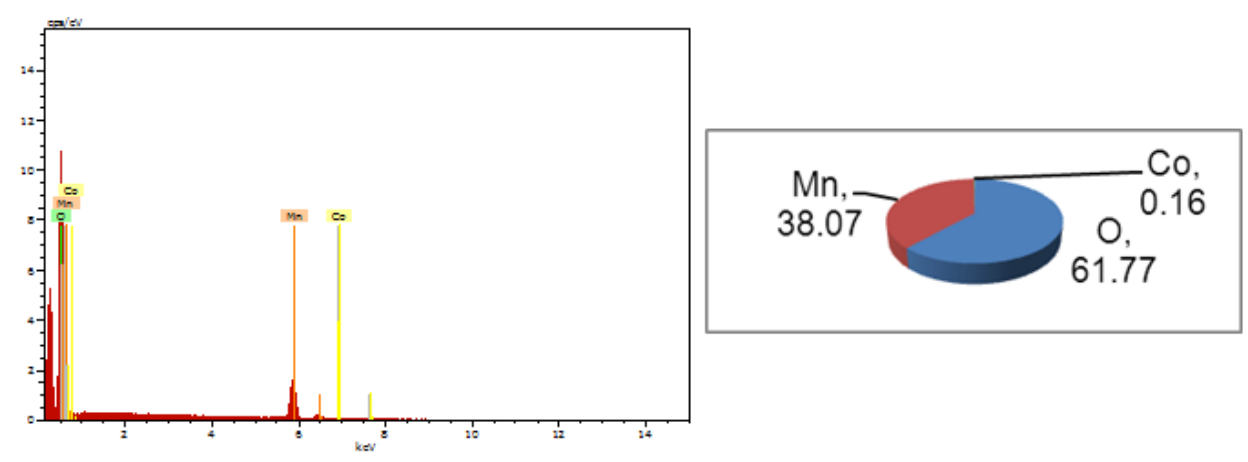



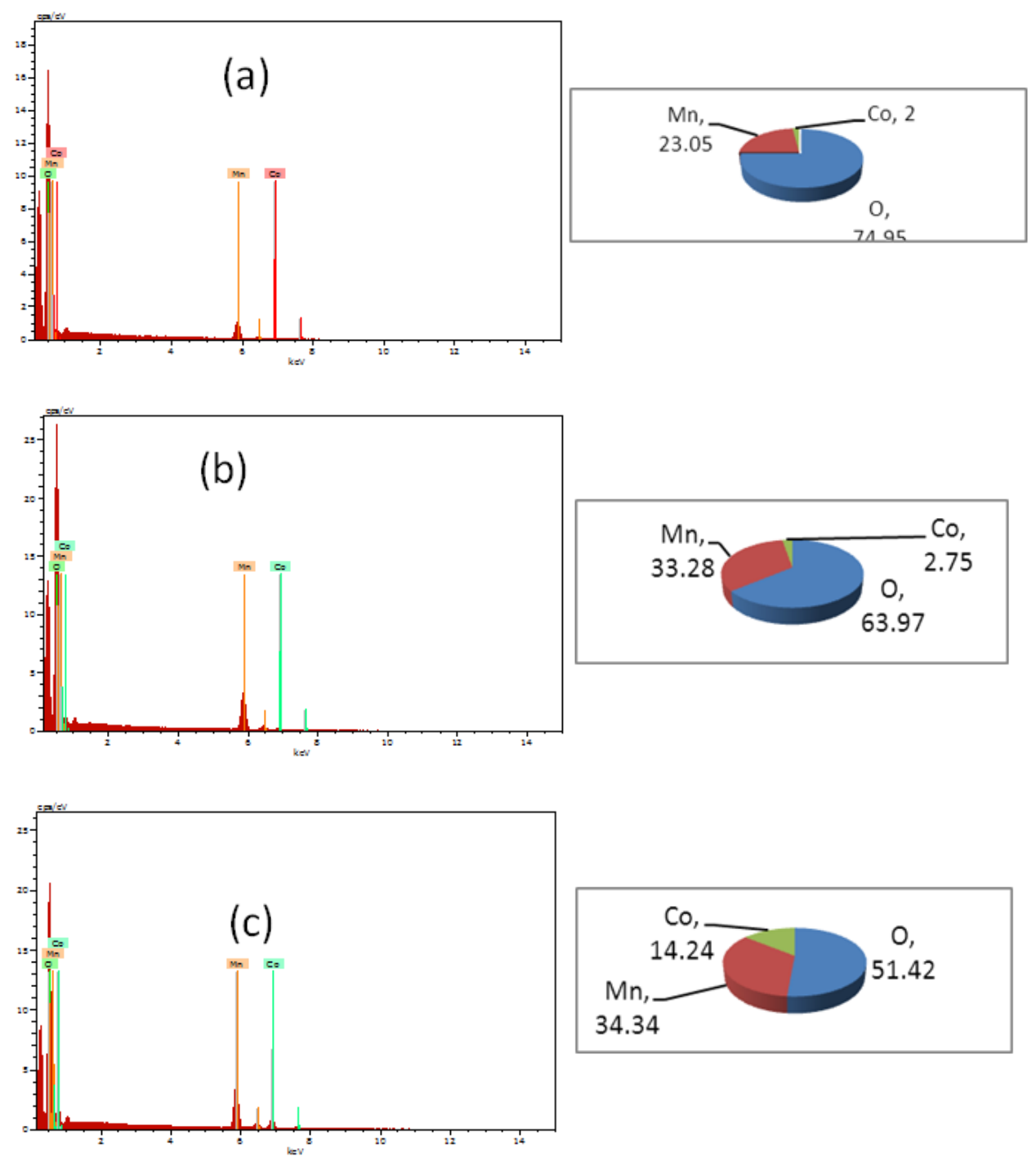

Fig.4 -EDAX spectrum of (a) 2\% Cobalt doped Manganese Oxide, (b) 4\% Cobalt doped Manganese Oxide, (c) $6 \%$ Cobalt doped Manganese Oxide \& (d) $8 \%$ Cobalt doped Manganese Oxide

\subsection{UV-Visible Spectral Analysis:}

UV-Visible spectra illustrate that pure Manganese Oxide nanoparticles exhibits maximum absorbance at $376 \mathrm{~nm}$. Fig.5 (a) shows the Ultraviolet -Visible absorbance spectra of pure $\mathrm{MnO}_{2}$ nanoparticles. Direct band gap of $\mathrm{MnO}_{2}$ is determined by fitting the absorption data to the transition equation $\boldsymbol{\alpha} \boldsymbol{h} \boldsymbol{v}=\boldsymbol{A}\left(\boldsymbol{h} \boldsymbol{v}-\boldsymbol{E}_{g}\right)^{2}$. Optical band gap energy $E_{\mathrm{g}}=3.15 \mathrm{eV}$ was obtained by extrapolating the linear part of the curve $(\alpha \mathrm{h} v)^{2}$ versus photon Energy hv (eV) known as Tauc plot in Fig.-5(b).

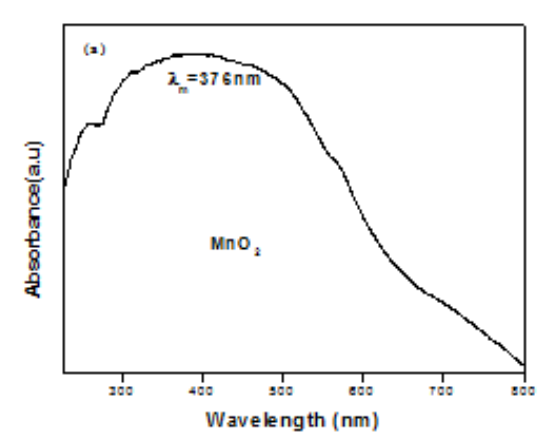

Fig.5-(a) UV-Visible absorption spectrum of pure $\mathrm{MnO}_{2}$ nanoparticles

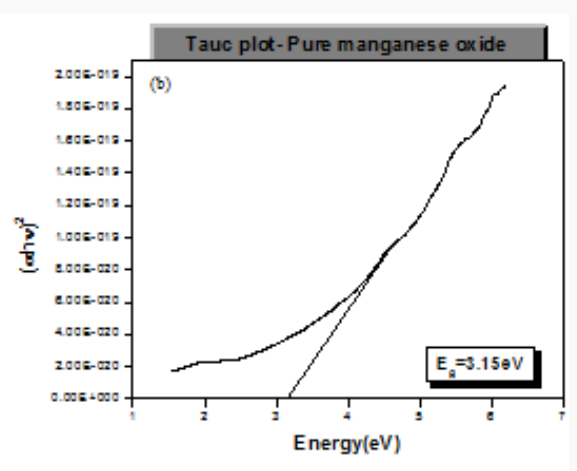

(b) (ahv) ${ }_{2}$ versus Energy (eV) 
The Cobalt doped Manganese oxide nanoparticles acquire blue shift from the pure $\mathrm{MnO}_{2}$ nanoparticles and absorption wavelengths of dopant concentration $2 \%, 4 \%, 6 \%$ and $8 \%$ are $280 \mathrm{~nm}, 273 \mathrm{~nm}, 270 \mathrm{~nm}$ and $265 \mathrm{~nm}$ from UV-Vis absorption spectra in Fig.6-(a).

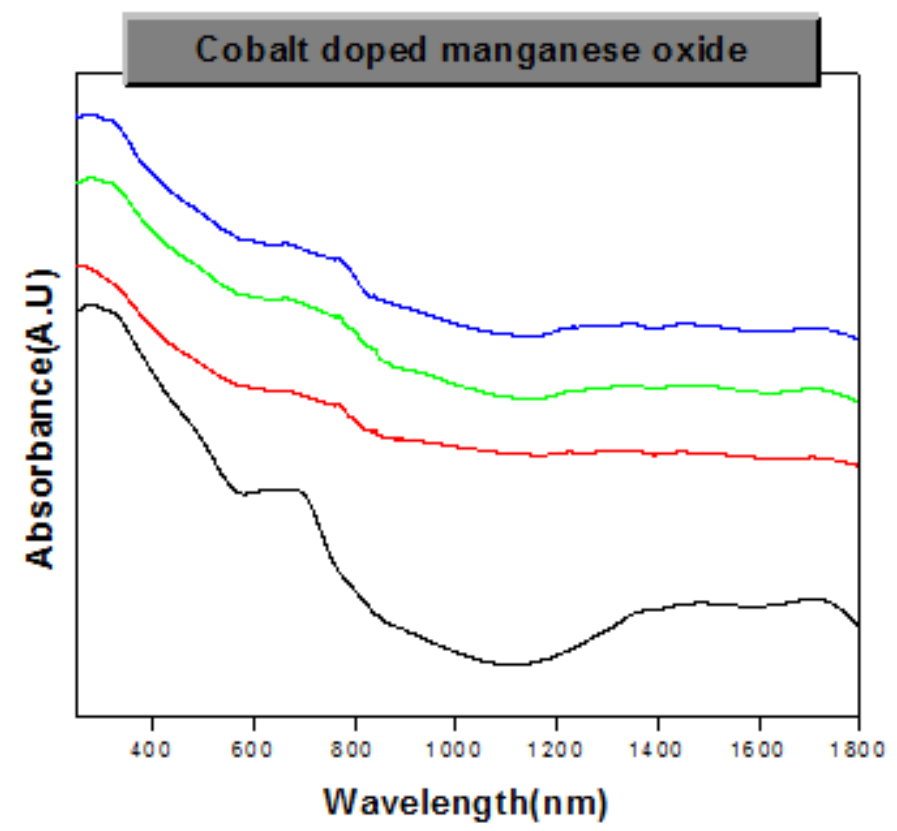

Fig.6- UV-Visible absorption spectrum of Cobalt doped $\mathrm{MnO}_{2}$ Nano particles

From the Tauc plot band gap energy of Cobalt doped Manganese Oxide nanoparticles are measured $4.43 \mathrm{eV}$, $4.545 \mathrm{eV}, 4.496 \mathrm{eV}$ and $4.682 \mathrm{eV}$ as shown in Fig.-7 (a), (b), (c) \& (d).
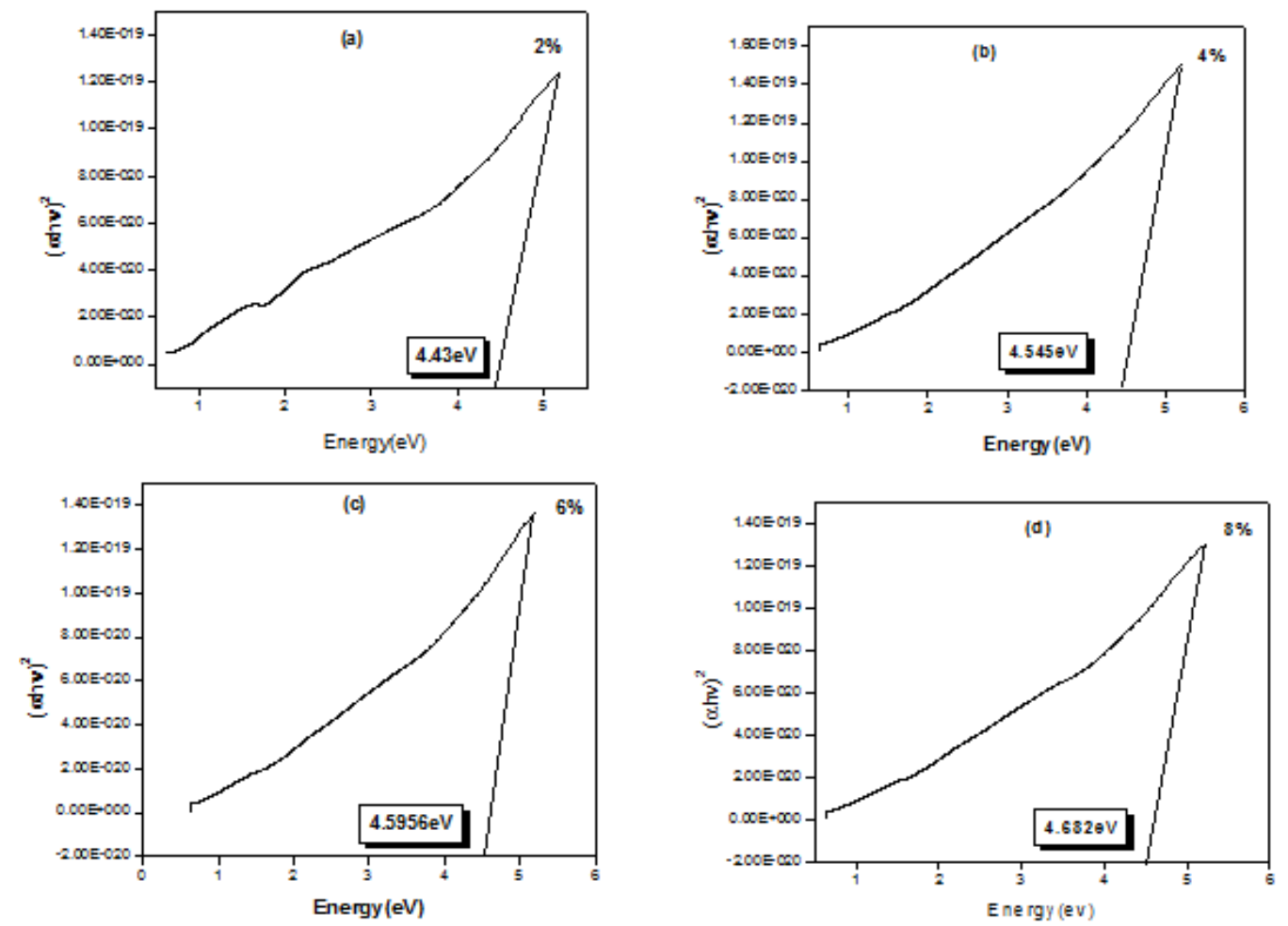

Fig.7- Tauc plot of (a) 2\% Cobalt doped Manganese Oxide, (b) 4\% Cobalt doped Manganese Oxide, (c) 6\% Cobalt doped Manganese Oxide \& (d) $8 \%$ Cobalt doped Manganese Oxide 


\subsection{Magnetic Properties:}

As prepared $\mathrm{MnO}_{2}$ nanoparticles are intermediate product [4].The magnetic properties of synthesized $\mathrm{MnO}_{2}$ nanoparticles are characterized by VSM and obtained the hysteresis loops are shown in Fig 8.The M-H curve is linear with low coercivity (10.767gauss). Due to the amount of increasing the dopant concentration, the size of the particle decreases with greater coercivity. The nanosystem gain their single domain character with their spins are aligned parallel.The magnetic parameters are recorded in Table2.
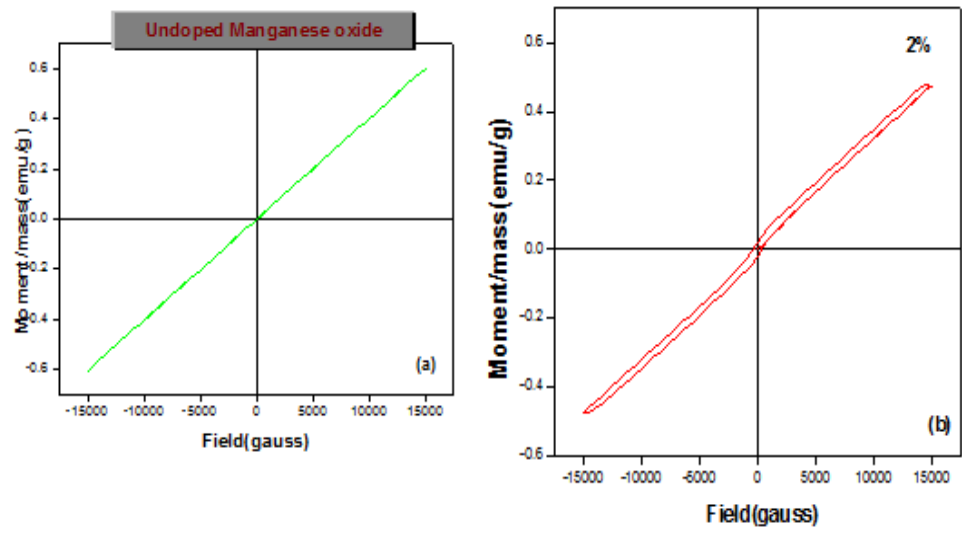

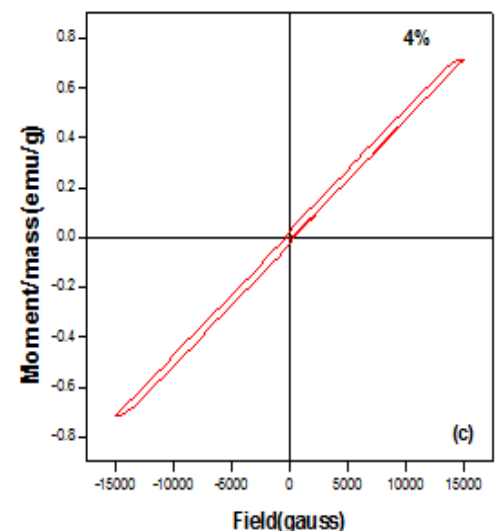

Field(gauss)
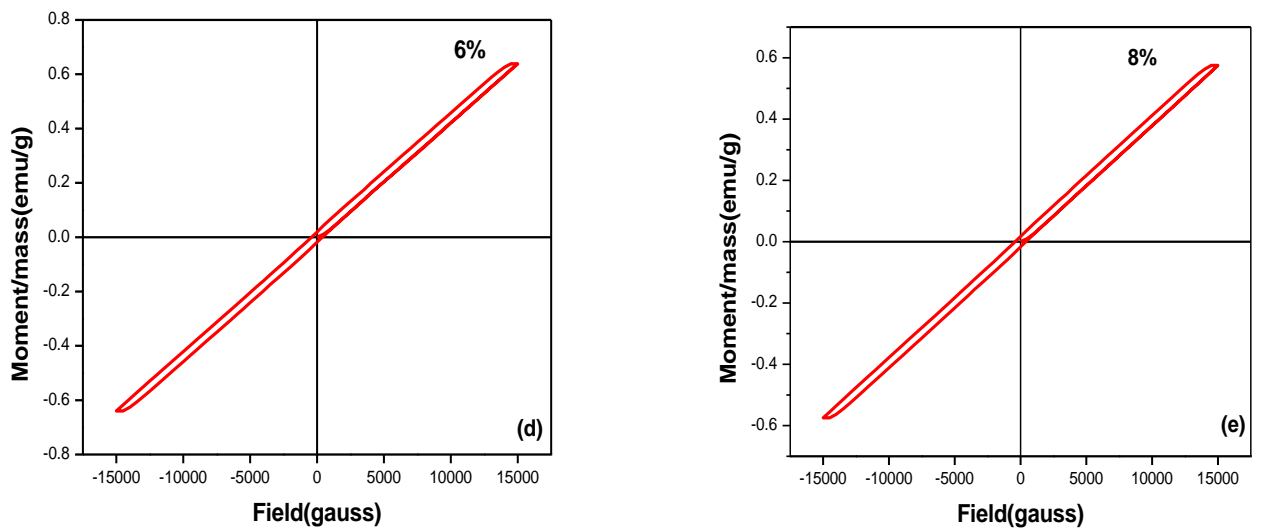

Fig.8- Magnetic Hysterisis loop of (a) Undoped Manganese Oxide (b) 2\% Cobalt doped Manganese Oxide, (c) 4\% Cobalt doped Manganese Oxide (d) 6\% Cobalt doped Manganese Oxide; (e) 8\% Cobalt doped Manganese

Oxide

Table2: Magnetic Parameters From Hysteresis Loop

\begin{tabular}{lllll}
\hline SAMPLE & $\begin{array}{l}\text { COERCIVITY } \\
\text { (gauss) }\end{array}$ & $\begin{array}{l}\text { MAGNETIZATION } \\
(\mathrm{memu})\end{array}$ & $\begin{array}{l}\text { RETENTIVITY } \\
(\mu \mathrm{emu})\end{array}$ & $\begin{array}{l}\text { SQUARENESS RATIO } \\
(\mathrm{SQR}) \\
\left(\mathrm{M}_{\mathrm{r}} / \mathrm{M}_{\mathrm{s}}\right)\end{array}$ \\
\hline $\mathrm{MnO}_{2}$ & 10.767 & 23.489 & 17.485 & 0.7443 \\
$\mathrm{MnO}_{2}: \mathrm{Co}(\mathbf{0 . 0 2})$ & 393.61 & 23.786 & 956.23 & 0.0402 \\
$\mathrm{MnO}_{2}: \mathrm{Co}(\mathbf{0 . 0 4})$ & 398.71 & 35.712 & 108.16 & 0.0302 \\
$\mathrm{MnO}_{2}: \mathrm{Co}(\mathbf{0 . 0 6})$ & 401.75 & 30.703 & 878.03 & 0.0285 \\
$\mathrm{MnO}_{2}: \mathrm{Co}(\mathbf{0 . 0 8})$ & 408.33 & 29.890 & 861.38 & 0.0288 \\
\hline
\end{tabular}

\section{Discussion}

The particle size reduces with increase of dopant concentration. The Bragg reflections from (200), (110), (210), (310), (011), (400), (211) and (320) planes which corresponds to $\mathrm{MnO}_{2}$ (JCPDS 82-2169).The value of crystallite size of pure manganese oxide is equal to $39 \mathrm{~nm}$ [5]. Due to the smaller shift in angle of diffraction, the lattice parameters also slightly varied $( \pm 10 \%)$.

The SEM image shows that having spherical geometry. It consists of irregular particles with a variety of pores due to the evolution of large amount of gases that are formed as by product during synthesis [6].The size of the nanoparticles $30 \mathrm{~nm}$ to $40 \mathrm{~nm}$ is very agreed with the SEM analysis by Image $\mathbf{J}$ software. 
A close interpretation of the FTIR spectra reveals metal oxides generally give absorption bands below $1000 \mathrm{~cm}^{-1}$ that arise from interatomic vibrations of nanoparticles. It represents the band at $533.35 \mathrm{~cm}^{-1}$ and $666.73 \mathrm{~cm}^{-1}$. This executed vibration bands could be assigned as metal - oxygen (Mn-O) bending vibrations [7]. The vibration band at $478.35 \mathrm{~cm}^{-1}$ and $486.06 \mathrm{~cm}^{-1}$ shows the existence of Co-O stretching vibration mode. The intensity of Co-O stretching bond increases with increasing the concentration of Cobalt [8]. The wavenumber $1392.22 \mathrm{~cm}^{-1}$ and $1342.46 \mathrm{~cm}^{-1}$ represents $\mathrm{O}_{2}$ stretching frequency [9]. A stretching frequency at $3394.72 \mathrm{~cm}^{-1}$ and a weak asymmetric band at $1566.20 \mathrm{~cm}^{-1}$ support the presence of hydroxyl (-OH) group due to the absorption of water by nanoparticle during the sample preparation[10]. The wavenumber $2299.15 \mathrm{~cm}^{-1}$ and $2376.30 \mathrm{~cm}^{-1}$ assigned to the $\mathrm{CO}_{2}$ mode. The $\mathrm{CO}_{2}$ modes are present in the FTIR spectra not owing to the serious contamination in Co doped $\mathrm{MnO}_{2}$ but these modes due to atmospheric $\mathrm{CO}_{2}$ in the samples. Samples might have trapped some $\mathrm{CO}_{2}$ from the atmosphere during FTIR characterization might have given such modes [11\&12].

EDAX allows the elemental composition of the specimen to be measured. The energy of the X-rays emitted from a specimen can be measured by an energy-dispersive spectrometer. EDAX spectrum shows the presence of dominant elements Manganese, Oxygen, and Cobalt.

$\mathrm{MnO}_{2}$ nanoparticles have very high absorbance in the visible region. Cobalt doped $\mathrm{MnO}_{2}$ nanoparticles absorption peaks shift towards smaller wavelength (higher band gap energy) as their crystal size decreases. So the prepared samples are used in solar cells and photo-catalysts [13].

The addition of ferromagnetic material like Cobalt with anti-ferromagnetic material $\mathrm{MnO}_{2}$ results ferromagnetic nature. The field necessary to reduce the net moment to zero is defined as the coercive field $\left(\mu_{o} H_{c}\right)$ or anisotropy field. The coercivity increases with dopant concentration increases but retentivity is decreases. The Cobalt doped Manganese oxide nano particles exhibited clear hysteresis showing typical ferromagnetic behavior that is 'positive spin interactions' when the spins are aligned in one single direction, having non-zero coercivity and retentivity. The area of the hysteresis curve is very small. This shows that the material formed is of soft magnetic material which is useful in magnetic memory devices [14]. In general large SQR values are desired for recording medium. Hc is a very complicated parameter for magnetic films and is related to the reversal mechanism and the magnetic microstructure, i.e., shape and dimensions of the crystallites, nature of the boundaries, and also the surface and initial layer properties, etc.

\section{Conclusion}

$\mathrm{MnO}_{2}$ and Cobalt doped $\mathrm{MnO}_{2}$ Nanoparticles were successfully synthesized by the solvothermal method employing microwave irradiation. XRD, SEM FTIR, EDAX UV-Vis and VSM studies are carried out. The XRD spectrum shows the samples are in single phase. The pure and Cobalt doped nanoparticles are having orthorhombic structure. The particle sizes of synthesized pure $\mathrm{MnO}_{2}$ nanoparticles are $38 \mathrm{~nm}$. For Co doped $\mathrm{MnO}_{2}$ samples the particle size decreases. The FTIR spectra and EDAX spectra indicate the formation of Pure and Co doped $\mathrm{MnO}_{2}$ nanoparticles. UV-Vis spectra illustrates that Cobalt doped Manganese oxide nanoparticles acquire blue shift from the bulk $\mathrm{MnO}_{2}$ nanoparticles (i.e.) $3.15 \mathrm{eV}$. Energy band gap increases with Cobalt concentration due to the effect of quantum confinement. The magnetic studies reveal the room temperature ferromagnetic behaviour of Cobalt doped $\mathrm{MnO}_{2}$ nanoparticles. The prepared Samples can be used as soft electro-magnetic materials. Then, focus on electrical studies for further analysis, the most promising electrode material Manganese oxide in lithium-ion batteries and super capacitor applications with the effect of dopant concentration.

\section{Acknowledgement}

I would like to express my special thanks of gratitude to my guide Dr.S John Kennady Vethanathan as well as my co-guide Dr.S Perumal.My thanks really go to my friends who helped me a lot in finalizing this article within the time frame.

\section{References}

[1]. Xiaodi Liu, Changzhong Chen, Yiyang Zhao and Bin Jia 2013 A Review on the Synthesis of Manganese Oxide Nanomaterials and their Applications on Lithium-Ion Batteries Journal of Nanomaterials Volume, Article ID 736375:7pages

[2]. Pugazhvadivu K.S,.Ramachandran K and K.Tamilarasan 2013 Synthesis and Characterization of Cobalt doped Manganese oxide nanoparticles by chemical route Physics procedia $49: 205-216$

[3]. Xiong Zhang, Xianzhong Sun, Haitao Zhang, Dacheng Zhang, Yanwei Ma 2013 Microwave-assisted reflux rapid synthesis of MnO2 nanostructures and their application in supercapacitors Electrochimica Acta 87: 637-644

[4]. Shuping Zhang, Wei Liu, Jie Ma and Yan Zhao 2010 A facile low-temperature route for preparing monodisperse Mn3O4 nanopolyhedrons from amorphous MnO2 nanoparticles NSTI - Nanotech,ISBN 978-1-4398-3401-5 Vol 1

[5]. Harish Kumar, Manisha and Poonam Sangwan 2013 Synthesis and Characterization of MnO2 nanoparticles using Co-precipitation Technique International journal of Chemistry and Chemical Engineering ISSN 2248-9924 Volume 3 Number 3: pp.155-160

National Conference on Current Advancements in Physics $3^{\text {rd }} \& 4^{\text {th }}$ February 2017 41 | Page

Department of Physics, St. John's College, Palayamkottai-627 002, Tamilnadu, India. DOI 10.9790/4861-17002013442 
[6]. Pradeep kumar BM, Sriram Karikkat, Hari Krishna R, Udayashankara TH, Shivaprasad KH and Nagabhushana BM 2013 Synthesis and Characterization of Nano MnO2 and its adsorption characteristics over an Azo Dye.RESEARCH AND REVIEWS: JOURNALS OF MATERIAL SCIENCES Volume 2 Issue 1

[7]. SONG Rui, WANG Hong-jun and FENG Shou-hua 2012 Solvothermal Preparation of Mn3O4 Nanoparticles and Effect of Temperature on Particle Size CHEM.RES.CHENESE UNIVERSITIES 28(4):577-580

[8]. Anjali A.Athawale, Megha Majumdar, Hema singh and K.Navinkiran. 2010 Synthesis of cobalt oxide nanoparticles /fibres in Alcoholic medium using gamma ray technique. Defence science journal, vol-60,No.5: pp.507-513

[9]. Muthuchudarkodi R.R. Vedhi, C Appl-nanoscience 2015 Preparation and electrochemical characterization of manganese dioxide zirconia nanorods 5:481-491

[10]. Sherin J S,Thomas J K,Shiny Manoj Facile Synthesis and Characterization of Pyrolusite, $\beta$-MnO2 2015 Nano Crystal with Magnetic studies Volume 4 Issue 5 ISSN-2319-7560

[11]. Dole B,Mote V, Huse V,Purushotham Y,Lande M,Jodhav K, shah.S: Structural studies of Mn doped ZnO nanoparticles. Current Appl phys 2011,11: 762-766

[12]. Yao-Ming Hao, Shi-Yun Lou, Shao-Min Zhou, Rui-Jian Yuan, Gong-Yu Zhu and Bing Li, 2012 Structural ,optical and magnetic studies of manganese doped zinc oxide hierarchical microspheres by self -assembly of nanoparticles Nanoscale research letters

[13]. Majed Sharrouf, Ramadan Awad, Mohamad Roumie, Salem Marhaba 2015 Structural ,optical and room temperature study of Mn2O3 nanoparticles Materials Sciences and Applications, 6:850-859

[14]. Irfan Elahi,Rabab Zahira,Kiran Mehmood,Arifa jamil and Nasir Amin 2012 Co-precipitation synthesis, physical and magnetic properties of manganese ferrite powder,African journal of pure and Applied Chemistry vol6(1):pp1-5 